\title{
A case of trigeminal neuralgia due to dolichoectasia of the vertebrobasilar arteries
}

\author{
Fonseka CL, Tissera WAJN \\ National Hospital of Sri Lanka, Colombo, Sri Lanka.
}

Correspondence: Dr. Lakmal Fonseka

e-mail: fonseka.lakmal@gmail.com

\section{Introduction}

Trigeminal Neuralgia (TN) is a debilitating pain syndrome usually caused by demyelination of trigeminal sensory fibres within the nerve root. In most cases, the trigeminal nerve root demyelination involves the proximal portion of the root, resulting from compression by an overlying artery or vein (1). Dolichoectasia is a very rare cause of compression of trigeminal roots leading to TN. Currently there is less than 10 reported cases of vertebrobasilar dolichoectasias complicated by trigeminal neuralgia reported in literature (2).

Dolichoectasia is a rare but recognized vascular anomaly usually occurring in the vertebrobasilar system of intracranial arteries (3). It is characterized by elongated and tortuous arterial vessels subsequently leading to thrombosis, microembolisation, and brainstem compression, with or without aneurysm formation. It has been observed to cause isolated or combined brainstem/cranial nerve syndromes, cervico-medullary junction compression, transient or permanent motor deficits, cerebellar dysfunction, central sleep apnoea and hydrocephalus. It has also given rise to ischaemic and hemorrhagic stroke and subarachnoid hemorrhage $(4,5)$. Although, it is not commonly observed, it should be considered as a cause which could lead to significant neurological morbidity and mortality.

We report a patient with dolichoectasia of vertebrabasilar system presenting with debilitating $\mathrm{TN}$, who subsequently improved with local anesthetic injections through radiographic guidance.

\section{Case report}

We present a 70 years-old male who presented with episodic left sided hemi-facial pains for the last two months. He is a patient with hypertension with a fairly good control. His episodes of pain started to occur once or twice a day initially. He described an excruciating electric shock like pain over the V1 and V2 areas of the trigeminal nerve dermatomes, which predominantly got precipitated by washing his face. Gradually, his pain worsened and frequency also increased to 10-20 episodes per day. During the exacerbations he took paracetamol, tramadol, carbamazepine and also oral morphine without much benefit. He was feeling depressed and anxious and avoided any activity which could precipitate another attack, including washing face and chewing food. He did not consent for very invasive surgical procedure.

We investigated him with non-contrast and contrast CT scan (Figure 1) and also with an angiogram of cerebral vessels (Figure 2). It showed a Vertebrobasilar dolichoectasia without any aneurysms or arterio-venous malformations. His basic blood investigations were all normal.

As the pain was resistant to maximum pain relief medication, including subcutaneous regular dose of Morphine, we arranged an injection of a local anesthetic agent (bupivacaine) combined with steroid through radiographic guidance, to the area around foramen ovale and repeated the injections weekly until maximum pain relief was achieved. With four weekly injections, the facial pains reduced significantly and we managed to maintain his pain relief with oral gabapentin and carbamazepine. Gradually, he could eat and wash his face and engage in usual activities of daily living. Within the last 2 years of clinic follow up, he required $2-3$ injections in two episodes of exacerbations, but was otherwise uneventful. 


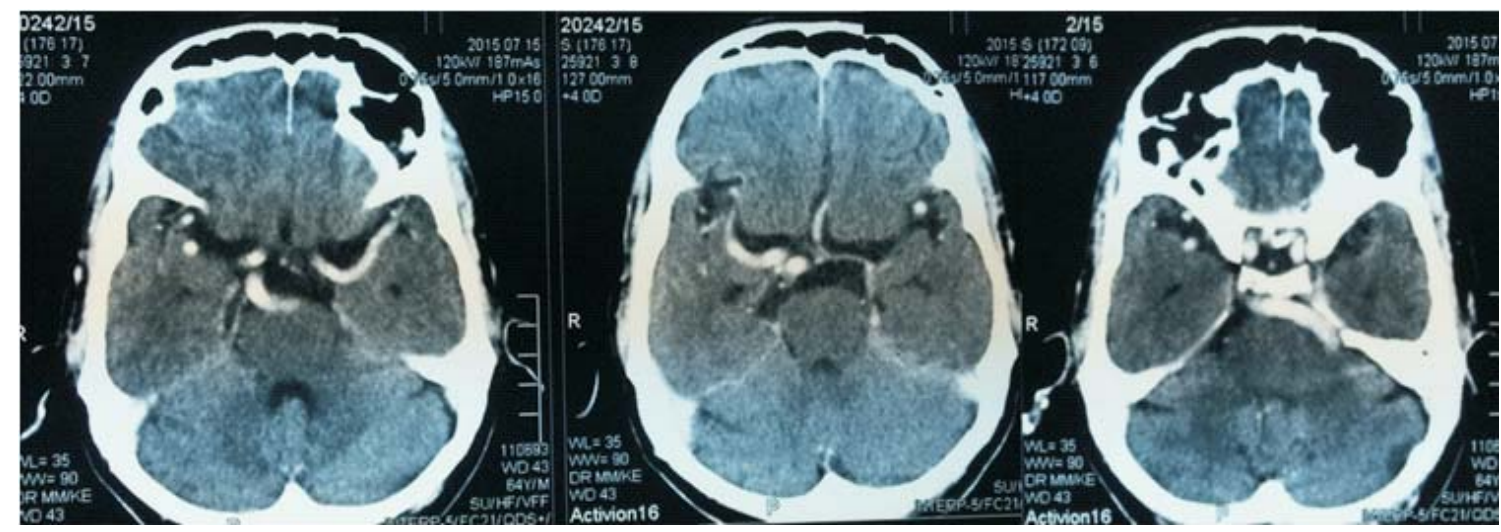

Figure 1: Contrast CT showing vertebrobasilar dolichoectasia

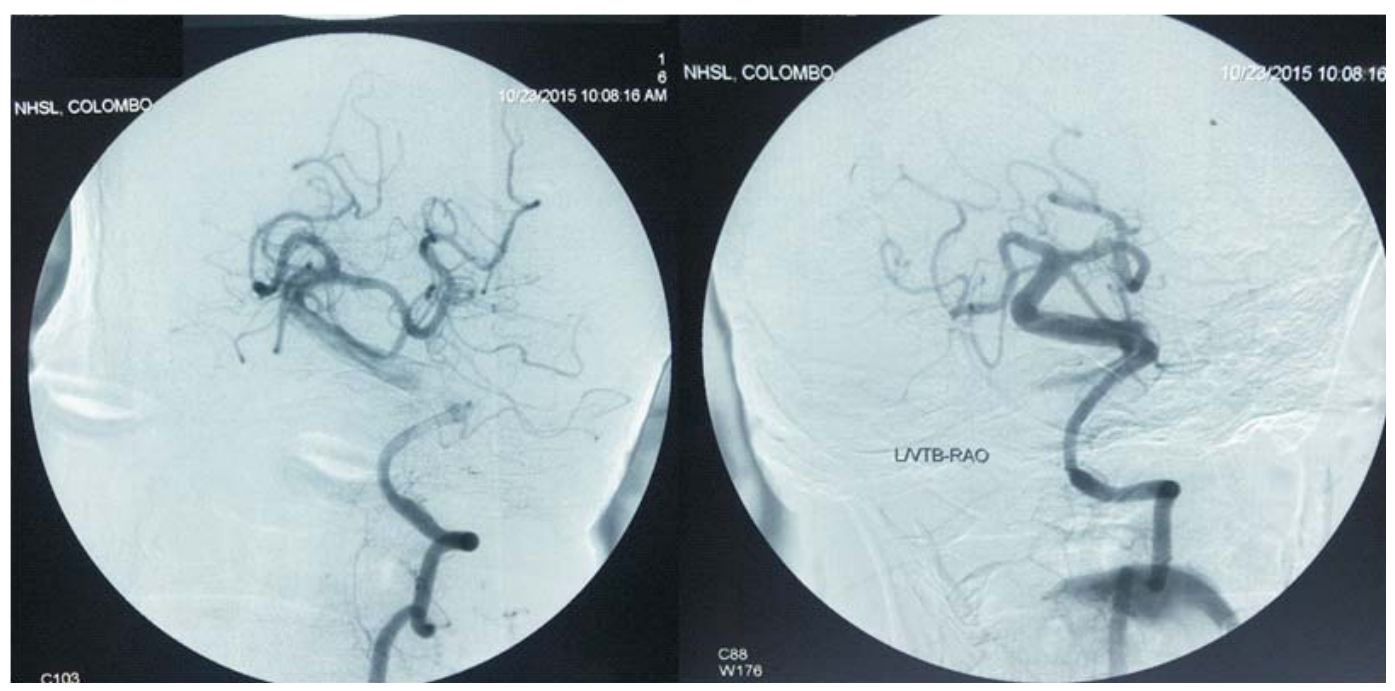

Figure 2: Angiography of cerebral vessels showing the elongated, tortuous arteries of circle of Willis. Vertebrobasilar dolichoectasia

\section{Discussion}

Dolichoectasia is a usually incidentally detected entity and is asymptomatic in majority. Vertebrobasilar dolichoectasia is an extremely rare entity with an expected incidence of $0.06-5.8 \%$. The disorder is more common in males and is seen usually in $6^{\text {th }}-7^{\text {th }}$ decade of life. The criteria for dolichoectasia include a diameter of more than $4.5 \mathrm{~mm}$, basilar length $>29.5$ $\mathrm{mm}$ or intracranial vertebral artery length $>23.5 \mathrm{~mm}$ and deviation of the ectatic vessel of more than $1 \mathrm{~cm}$ beyond the shortest normal course (3).
Compressive etiology is the most common way leading to TN. So, microvascular decompression is considered the preferred treatment for medically resistant cases of TN and gamma knife therapy has emerged as a recommended alternative, particularly in elderly patients with comorbidities (8). However, over 30 years of follow up, the incidence of recurrence after microvascular surgery has been reported to range from 3 to $30 \%(9)$.

Our patient did not consent for surgery, so that we had to go ahead with an alternative mode of treatment. Therefore, local anesthetic injection combined with steroids was attempted as an option. 
With this strategy, he showed a long lasting response and thereafter, he only necessitated few injections in the two exacerbations that he had over the next 2 years of follow up.

\section{Conclusions}

Dolichoectasia is a very rare but a noteworthy cause for Trigeminal Neuralgia (TN). It is important to be vigilant about the compressive causes which may lead to TN, especially when they are resistant to oral medication. When managing resistant cases, it would be beneficial to try less invasive methods such as, Trigeminal nerve blocks with local anesthetic and administering pain relief for neuropathic pain in patients who are not willing for invasive surgery.

\section{References}

1. Love S, Coakham HB. Trigeminal Neuralgia: pathology and pathogenesis. Brain 2001 Dec; 124(12): 2347-60.

2. Kraemer JL, Pereira Filho A de A, David Gd, Faria M de B. Vertebrobasilar dolichoectasia as a cause of trigeminal neuralgia: the role of microvascular decompression. Case report. Arq Neuropsiquiatr 2006 Mar; 64(1): 128-31.
3. Gutierrez J, Sacco RL, Wright CB. Dolichoectasia - an evolving arterial disease. Nat Rev Neurol 2011; 7: 41-50.

4. Wolters FJ, Rinkel GJ, Vergouwen MD. Clinical course and treatment of vertebrobasilar dolichoectasia: a systematic review of the literature. Neurol Res 2013 Mar; 35(2): 131-7.

5. Passero SG, Rossi S. Natural history of vertebrobasilar dolichoectasia. Neurology 2008 Jan 1; 70(1): 66-72.

6. Prasad S, Galetta S. Trigeminal neuralgia: historical notes and current concepts. Neurologist 2009 Mar; 15(2): 87-94.

7. Lee SH, Levy EI, Scarrow AM, Kassam A, Jannetta PJ. Recurrent trigeminal neuralgia attributable to veins after microvascular decompression. Neurosurgery 2000; 46: 356. 